\section{Motor behaviour modifications after a stroke in a patient with Huntington's disease}

Sir: We describe the effects induced by an ischaemic focal brain lesion on the choreic movements of a patient with Huntington's disease.

Extrapyramidal movement disorders often improve or disappear after damage to the corticospinal motor system. However, when the change in motor behaviour symmetrically involves both sides of the body, they cannot usually be ascribed to a focal unilateral lesion of the corticospinal motor tract. Changes in levels and turnover of catecholamines in the different phases of a stroke have been reported by several authors. $^{1-s}$ We suggest that bilateral changes in chorea after stroke may be due to an alteration of the neurotransmittersystem induced by the stroke.

On 2 October, 1982, a 61 year-old-man with Huntington's chorea was admitted after the sudden onset of a right hemiparesis. He had been followed for 3 years for his chorea, which appeared 5 years earlier. During the last 10 months the patient was treated with haloperidol (2 $\mathrm{mg} /$ day). Two months before the stroke clinical examination revealed: $\mathrm{IQ}=85$, measured by means of Wechsler Adult Intelligence Scale; chorea severity $=3 \cdot 5$, evaluation as indicated by Frattola et al. ${ }^{\circ}$ There were no cerebellar or pyramidal symptoms, nor alteration of mood.

In the morning of 1 October, the patient suddenly developed a right-sided hemiparesis, with paresthesiae and dysphasia. These symptoms lasted for about 2 hours; after this period they gradually cleared and resolved almost completely in the following 6-8 hours.

At the time the neurological symptoms appeared, the patient also had marked bilateral exacerbation of his chorea (almost twice the usual, as reported by various relatives). This worsening of chorea lasted throughout the day, and was so pronounced it was difficult for the patient to walk or to sleep. The patient remained at home and no medical examination was done at this day. The following morning, on awakening, he had a right hemiparesis together with complete bilateral disappearance of chorea.

On admission he had a right sided hypotonic hemiparesis with cortical sensory deficit, both more marked in the face and upper limb. The tendon reflexes were increased on the right side, with a right extensor plantar. The overall neurological deficit score (evaluated according to Prescott $e t a l^{7}$ ) was eight. Choreic movements were almost completely absent at this time. The conscious level remained normal. The blood pressure was 125/80 mm Hg; EKG and the results of routine laboratory investigations were normal. A computed tomographic (CT) brain scan revealed a small deep periventricular infarct in the white matter of the left hemisphere and generalised cortical atrophy (fig). EEG revealed diffuse theta waves. The patient was treated with dexamethasone (12 $\mathrm{mg} /$ day) for 7 days. His neurological deficits gradually improved, but one week after the onset of the stroke chorea began to reappear in the face and four limbs, increasing in severity and reaching a score of five after 14 days. For this reason haloperidol treatment, stopped 12-18 hours after the onset of the stroke, was started again, and there was a decrease in the chorea severity. At the end of the first month a second CT brain scan revealed a reduction of the ischaemic area. Four vessel angiography was normal.

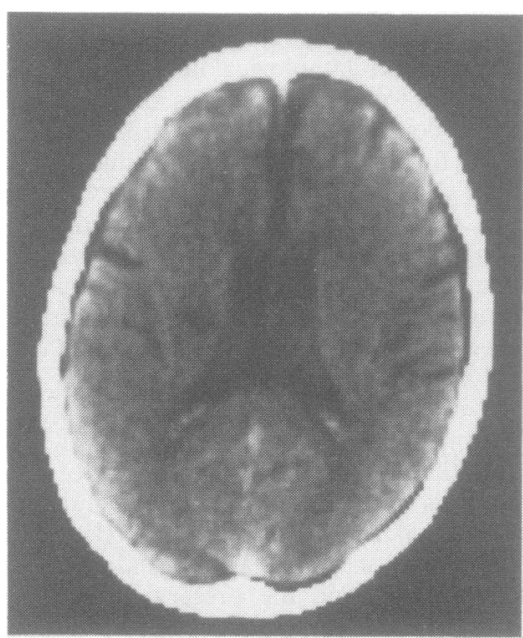

Fig Unenhanced CT scan showing a small deep periventricular infarct in the white matter of the left hemisphere.

It is not easy to find a clear explanation for the mechanisms leading to the fluctuations of the chorea, and the relation to the ischaemic lesion observed in our patient. In particular, the bilateral increase of choreic movements during the transient phase of the stroke and their subsequent disappearance can hardly be explained by damage to a well defined unilateral neuronal popula- tion. Several studies have shown a $\bar{Z}$ increased release of catecholamines aftef an ischaemic cerebral event. In animals? dopamine content increases in the braif during the early phase after stroke, while if decreases a few days after the lesion. ${ }^{-}$(5) The turnover of biogenic amines probabls may change in a similar way in the huma brain: the human cerebrospinal fluid con tent of catecholamines increases in the first day after the stroke. ${ }^{8}$ Since catecholamine are released in a biologically active form by infarcted neurons, their massive output could be responsible both for the progres sion of the ischaemic process and for those motor disorders observed in aminals in th early post-ischaemic period. ${ }^{2} \mathrm{~s}$

Abnormal involuntary movements are usually ascribed to overactivity of brai $\vec{\Phi}$ monoamine systems, in particular dopamine, at the level of the basal gangliaw We may speculate that in the reported case the marked exacerbation of chorea during the first hours of the ischaemia may have been due to increased cerebral neurotransmitters; the hyperkinetic syndrom disappeared almost completely after the next 12-24 hours, possibly in parallel $\mathrm{Ji}^{1} \mathrm{fl}$ brain catecholamine depletion. Finallyọ ho chorea began to reappear one week fifte the stroke, thus indicating restored finc tional equilibrium in the neurotransminte dinamics. It is important to note thy although the lesion was localised in a sign area deep in the left hemisphere, chatg. in chorea appeared on both sides ofoth body. In animals the neurochemical consequence of induced ischaemic lesions ato diffused in areas which are not directify affected by ischaemia and even affect the contralateral hemisphere. ${ }^{135}$ The onset generalised choreiform movements h⿱亠 been reported in transient cerebra ischaemia and in chronic subdur haematomas, ${ }^{10}$ but the pathogenetie mechanisms underlying the appearance this clinical syndrome are still unknow. The possibility should be considered that changes in neurotransmitter regulation may offer an explanation of those altera tions in the motor and affective behaviour which cannot be interpreted as the result of a focal brain lesion. ${ }^{11}$

LODOVICO FRATTOLA MARIA SBACCI MARCO TRABUCCH Neurological Clinie, University of Milar

Bassini Hospital, via Gorki 5 军. 20092-Cinisello Balsamo 


\section{References}

' Kogure KP, Scheinberg A, Matsumoto R Busto R, Reinmuth O. Catecholamines in experimental brain ischemia. Arch Neurol 1975;32:21-4.

${ }^{2}$ Moskowitz MA, Wurtman RJ. Acute stroke and brain monoamines. In: Scheinberg P, ed. Cerebrovascular Diseases. New York: Raven Press, 1976: 153-66.

${ }^{3}$ Bralet J, Beley P, Bralet AM. Catecholamine levels and turnover during brain ischemia in the rat. J Neural Transmission 1980; 48: 143-55.

${ }^{4}$ Harrison MJG, Marsden CD, Jenner P. Effect of experimenatal ischemia on neurotransmitter amines in the gerbil brain. Stroke 1979; 10: $165-8$.

${ }^{5}$ Robinson RG. Differential behavioural and biochemical effects of right and left hemispheric cerebral infarction in the rat. Science 1979; 205: 705-10.

- Frattola L, Albizzati M, Spano PF, Trabucchi M. Treatment of Huntington's chorea with bromocriptine. Acta Neurol Scand 1977; 56:37-45.

${ }^{7}$ Prescot RJ, Garraway AJ. Predicting functional outcome following acute stroke using a standard clinical examination. Stroke 1982;13:641-7.

${ }^{8}$ Meyer JS, Welch KMA, Okamoto S, Shimazu K. Disordered neurotransmitter function. Demonstration by measurements of norepinephrine and 5-hydroxytryptamine in CSF of patient with recent cerebral infarction. Brain 1974;97:655-64.

${ }^{9}$ Margolin DI, Marsden CD. Episodic dyskinesias and transient cerebral ischemia. Neurology (NY) 1982;32:1379-80.

${ }^{10}$ Bae SH, Vates TS, Kenton EJ. Generalized chorea associated with chronic subdural hematomas. Ann Neurol 1980;8:449-50.

"Bloom FE. Neurotransmitters and CNS disease. The future. Lancet 1982;ii: 1381-5.

\section{Myelopathy secondary to metastatic car-} cinoid tumour

Sir: Metastasis of carcinoid tumour to the nervous system is uncommon ${ }^{1-5}$ and spinal cord compression from a metastasis has been reported on only one occasion. ${ }^{6}$ This is a report of a 56-year-old woman who presented with a thoracic myelopathy due to metastic carcinoid tumour.

She was admitted with a three month history of $T_{3}$ radicular pain. paraesthesiae in both legs, ataxia and falls. Three years previously she had had a laparotomy for small bowel obstruction due to an ileo-caecal carcinoid tumour. The bowel wall was infiltrated and similar tumour was found in the mesenteric lymph nodes. After operation an isotope liver scan was normal and screening for 5-hydroxyindole acetic acid (5-HIAA) was negative. On this admission examination showed an asymmetric spastic paraparesis without a sensory level. Palpation of $T_{3}$ and $T_{4}$ was painful. Plain radiography of the thoracic spine showed sclerosis of the body and pedicle of $T_{3}$. A myodil myelogram demonstrated an extradural block at the level of $T_{3}$. The CSF total protein was $4.0 \mathrm{~g} / 1$ without a pleocytosis.

A $\mathrm{T}_{2}-\mathrm{T}_{3}$ laminectomy was performed. After removal of the right $T_{3}$ pedicle a mass was seen in the anterior extradural space to the right of $T_{3}$ and in contact with the $T_{3}$ nerve root. A biopsy of this mass was taken and found to be carcinoid tumour (fig). During surgery there were wide fluctuations in blood pressure: the systolic pressure varying between 160 and $70 \mathrm{~mm} \mathrm{Hg}$; diastolic pressures between 105 and $40 \mathrm{~mm} \mathrm{Hg}$. These variations were directly related to manipulation of the tumour. On returning to the ward a urinary screen for 5-HIAA was negative.

After operation the patient was paraplegic. Treatment with radio-therapy was commenced on the eighth post-operative day and a total of 3570 rads were delivered in 16 sessions over 28 days to the affected part of the cord. The paraplegia slowly improved and three months later she was walking with the aid of a frame and leg caliper. Eleven months later she became jaundiced and at laparotomy multiple metastases were found in the liver. pelvis and mesentry. The urine now contained 5-HIAA. A cholecysto-jejunostomy was performed followed by abdominal radio-

therapy but she died 10 months later. 2 years 1 month after she presented with a paraparesis and 5 years after her original symptoms.

It is unknown why carcinoid tumours rarely metastasise to involve the central nervous system. This is the second reported case of carcinoid presenting as a spinal tumour. Both cases were middle aged women who presented with an asymmetric spastic paraparesis. In Kirkpatrick et al's patient the primary site was in the bronchus and the natural history of the disease was much longer. She was alive 10 years after her first neurological symptoms whereas this patient was dead within two and a half years. Although our patient's paraplegia improved following radiotherapy resolution of cord oedema or ischaemia may have contributed to her improvement.

Troublesome changes in blood pressure during surgery have been reported before ${ }^{7}$ and are thought to be due to the effect of vasoactive peptides released from the carcinoid tumour. These include serotonin. bradykinin. histamine. noradrenaline. dopamine. ${ }^{8}$ ACTH. $\beta$-endorphin and methoinine encephalin.' Prostaglandins have also been detected and they too have haemodynamic effects on the cardiovascular system. ${ }^{10}$

Although carcinoid tumours were thought to be resistant to irradiation improved prognosis with total abdominal radiotherapy has been reported." Management of carcinoids metastasising from

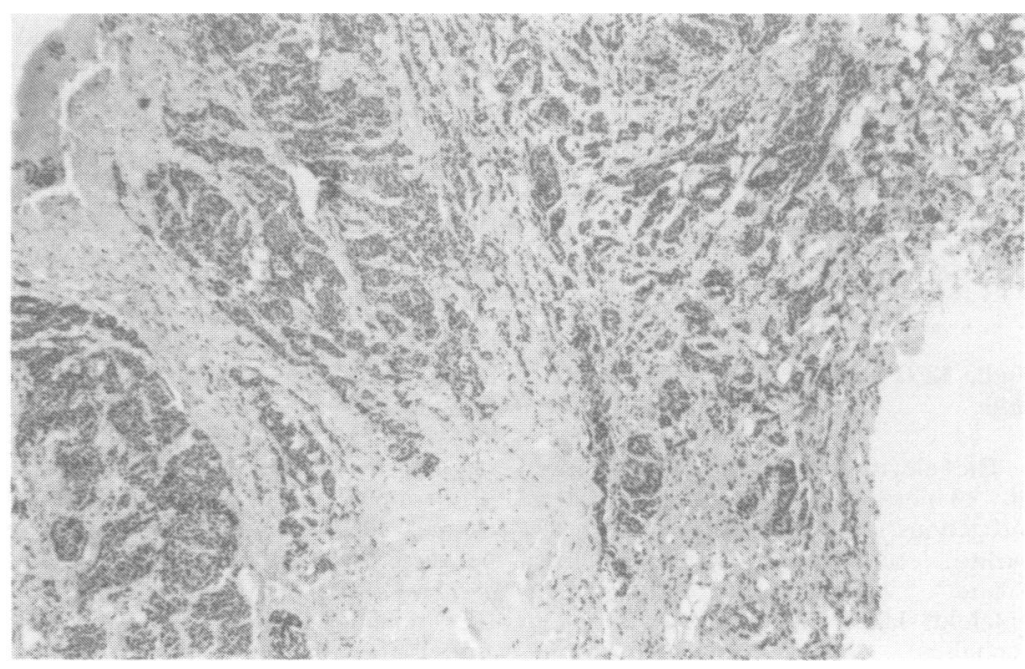

Fig Microscopic appearance of the $\mathrm{T}_{3}$ extradural mass showing carcinoid tumour $(\times 240)$. 